\section{Tauchen: Psychotrope Medikamente erhöhen nicht das Risiko von Stick- stoffnarkosen}

Krummel T et al. Psychotropic Drug Use in Recreational Scuba Divers and its Effect on Severe Narcosis. Int J Sports Med 2017; 38: 322 - 328

Tauchen mit einem Drucklufttauchgerät wird immer beliebter und zunehmend üben auch ältere, nicht vollständig gesunde Menschen den Sport aus. Damit steigt der Anteil von Sportlern, die unter einer Dauermedikation stehen, u.a. auch mit Anxiolytika oder Antidepressiva. Die Einnahme psychotroper Medikamente gilt aber eigentlich als Kontraindikation zum Tauchen, da sie das Risiko einer Stickstoffnarkose erhöhen sollen.

Allerdings gibt es dafür erstaunlicherweise kaum harte Belege. Daher haben nun französische Mediziner diese Frage näher untersucht.

Da eine interventionelle Studie dazu kaum durchführbar ist, hat die Arbeitsgruppe um Thierry Krummel eine Online-Umfrage in einer Gruppe von mehr als 11000 lizenzierten Sporttauchern angestellt. In dem Fragebogen ging es neben demografischen Daten um die Taucherfahrung, maximale Tauchtiefe, das Auftreten schwerer und lebensbedrohlicher Stickstoffnarkosen („Tiefenrausch“) bei einem Tauchgang und Einnahme von psychotropen Medikamenten (aufgeführt waren Schlafmittel, Anxiolytika, Antidepressiva, Neuroleptika, Antikonvulsiva und Opioide). Schwere Stickstoffnarkosen waren dabei definiert als das Auftreten von unangenehmen Symptomen, lebensbedrohliche Narkosen als solche, bei denen die Unterstützung durch einen zweiten Taucher notwendig wurde. Anschließend setzten die Wissenschaftler Stickstoffnarkosen und Einnahme der Medikamente zueinander in Beziehung.
Insgesamt 1608 Sporttaucher nahmen an der Umfrage teil (14,3\%). Die meisten waren erfahrene Taucher: $3 / 4$ von ihnen tauchten seit $>8$ Jahren, mehr als die Hälfte gab > 200 durchgeführte Tauchgänge an, und $3 / 4$ tauchten in eine Tiefe von $\geq 40 \mathrm{~m}$. Insgesamt 254 Teilnehmer $(15,2 \%)$ gaben die mindestens einmalige Einnahme eines psychotropen Medikaments an, etwa die Hälfte von ihnen nach Aufnehmen des Tauchsports ( $\mathrm{n}=$ 126). Über eine schwere Stickstoffnarkose berichteten 567 Taucher (35,3\%), über eine lebensbedrohliche Narkose 89 Taucher (5,5\%).

Dabei betrug die Häufigkeit einer schweren Stickstoffnarkose

- bei Tauchern mit Einnahme von psychotropen Medikamente 28,6\%,

- bei Tauchern ohne diese Medikamente $35,8 \%(p=0,1)$.

Ähnlich waren die Verhältnisse bei lebensbedrohlichen Narkosen (6,4 vs. $5,5 \% ; p=0,7)$. Auch bei Aufschlüsslung nach den oben angegebenen Medikamentenklassen fanden sich keine signifikanten Unterschiede zwischen den Gruppen.

Ebenso errechneten sich in der logistischen Regressionsanalyse keine Zusammenhänge von Stickstoffnarkose und Einnahme der psychotropen Medikamente. Die einzigen unabhängigen Risikofaktoren für das Auftreten waren die Anzahl der insgesamt durchgeführten Tauchgänge (Odds Ratio [OR] 2,012,83 mit zunehmender Anzahl gegenüber Tauchern mit <100 Tauchgängen) und die maximale Tauchtiefe (OR 1,04) $\mathrm{m})$. Diese Aussage blieb weitgehend unverändert, wenn nur lebensbedrohliche Narkosen berücksichtigt wurden: Hier waren weibliches Geschlecht (OR 0,60 für Männer) und maximale Tauchtiefe (OR 1,03/m) signifikante Risikofaktoren.
FAZIT

Nach diesen Daten scheint die Einnahme von psychotropen Medikamenten nicht per se mit einem erhöhten Risiko für eine Stickstoffnarkose beim Tauchen verbunden, so die Autoren. Tauchanfängern mit stabiler Erkrankung unter Einnahme eines solchen Medikaments müsse daher nicht unbedingt von dem Sport abgeraten werden, sondern sie sollten ihre Tauchtiefe unter Aufsicht erfahrener Kollegen ganz allmählich steigern, meinen Krummel et al. abschließend.

Dr. Elke Ruchalla, Bad Dürrheim 